\title{
Investigating the role of COQ8B in aortic smooth muscle cells.
}

\author{
Henry Stadler ${ }^{1}$, Stephanie Ware ${ }^{2}$, Benjamin Landis ${ }^{2}$ \\ ${ }^{1}$ Indiana University School of Medicine, ${ }^{2}$ Indiana University School of Medicine, \\ Departments of Pediatrics and Medical and Molecular Genetics
}

\section{Background and Hypothesis:}

Thoracic aortic aneurysm (TAA) is an aortopathy characterized by aortic enlargement and life-threatening complications such as aortic dissection and sudden cardiac death. Previous studies identified COQ8B as a candidate genetic modifier of TAA severity. COQ8B is important for mitochondrial biosynthesis of coenzyme $Q$, but its precise functions are not defined. We hypothesize that alteration of COQ8B influences TAA pathogenesis via energy and oxidant metabolism pathways.

\section{Experimental Design:}

Smooth muscle cells (SMCs) were cultured directly from leftover healthy aortic tissues acquired during cardiac transplant operations. At confluence of $50-70 \%$, cells were transfected with siRNA targeting COQ8B or a non-targeting negative control siRNA. Gene expression was measured using real-time quantitative polymerase chain reaction (RT-qPCR). Production of the reactive oxygen species hydrogen peroxide $\left(\mathrm{H}_{2} \mathrm{O}_{2}\right)$ was measured using the fluorescence-based Amplex Red Hydrogen Peroxide Assay (Invitrogen) in basal growth medium.

\section{Results:}

Expression of COQ8B decreased by approximately $75 \%$ to $85 \%$ at 48 hours following siRNA transfection compared with negative control. This was associated with approximately 1.5 fold upregulation of the SMC contractile gene CNN1 ( $p<0.05)$. Knockdown of COQ8B did not appear to alter $\mathrm{H}_{2} \mathrm{O}_{2}$ production measured at timepoints of 48 or 72 hours.

\section{Conclusion and Impact:}

Based on these preliminary data, decreased COQ8B expression appears to alter the contractile phenotype of SMCs but may not significantly influence extracellular levels of $\mathrm{H}_{2} \mathrm{O}_{2}$ under basal conditions. Exogenous activation of pathways important for TAA pathogenesis may be required to elucidate the role of COQ8B. Ultimately, this work may lead to improved clinical approaches. 